\title{
Divisible Designs with Dual Translation Group
}

\author{
Sabine Giese and Ralph-Hardo Schulz \\ To appear in DESIGNS, CODES and CRYPTOGRAPHY; the original \\ publication is available at www.springerlink.com

\begin{abstract}
Many different divisible designs are already known. Some of them possess remarkable automorphism groups, so called dual translation groups. The existence of such an automorphism group enables us to characterize its associated divisible design as being isomorphic to a substructure of a finite affine space.
\end{abstract}

AMS Classification: 05B05, 05B30, 20B25, 51N10

\section{Introduction}

Divisible designs (DDs) have been studied and constructed by many mathematicians. Since a variety of different DDs have been found, it is interesting to classify or characterize them.

In the present paper, we will deal with some DDs which have a remarkable automorphism group, called dual translation group, which provides a special characterization. DDs gained e.g. by a well known construction of A.G. Spera which is used in [13], or by a construction, called construction (A), given in [6], or by a generalization of both presented in [2], admit elementary abelian dual translation groups. As the main result, Theorem 3.1 shows that a DD which possesses such an automorphism group can be characterized as being isomorphic to a substructure of a finite affine space.

\section{Definitions and Examples}

Let a finite set $\mathcal{P}$ whose elements are called points be given. We denote a given equivalence relation on this point set by $R$ and call the induced equivalence classes point classes. We define $\mathcal{S}:=\{[x] \mid x \in \mathcal{P}\}$ as the set of all point classes with $[x]$ denoting the equivalence class containing the point $x \in \mathcal{P}$. A non-empty subset $B$ of $\mathcal{P}$ is called $R$-transversal if we have $|C \cap B| \leq 1$ for each point class $C \in \mathcal{S}$. Now, we recall the definition of a DD as follows. 
Definition 2.1 ( $t$-divisible design, $t$-DD, or simply DD) Let $t, s, k, \lambda_{t}$ be positive integers with

$$
t \leq k<v:=|\mathcal{P}| \text { and } t \leq \frac{v}{s} .
$$

A triple $\mathcal{D}=(\mathcal{P}, \mathcal{B}, \mathcal{S})$ is called a $t-\left(s, k, \lambda_{t}\right)$-divisible design (or $t-\left(s, k, \lambda_{t}\right)$ DD) if

(1) $\mathcal{B}$ is a set of $R$-transversal subsets of $\mathcal{P}$ with $|B|=k$ for all $B \in \mathcal{B}$;

(2) $|[x]|=s$ for all $x \in \mathcal{P}$;

(3) for every $R$-transversal $t$-subset $Y$ of $\mathcal{P}$, there exist exactly $\lambda_{t}$ elements of $\mathcal{B}$ containing $Y$.

The elements of $\mathcal{B}$ are called blocks.

Sometimes it is possible to deduce properties of a DD from its automorphism group (see e.g. translation DDs in [11], [12]). In this paper, we investigate DDs that admit a dual translation group which can be introduced in the following way.

Definition 2.2 Let $\mathcal{D}=(\mathcal{P}, \mathcal{B}, \mathcal{S})$ be a divisible design. We call the group $T$ a (full) dual translation group of $\mathcal{D}$, if

(1) $T \leq \operatorname{Aut} \mathcal{D}$

(2a) $T$ fixes all point classes of $\mathcal{D}$,

(2b) there exists a number $l$ such that every point class is the union of orbits under $T$ of length $l$,

(3) $T_{P} \neq T_{Q}$ for all $P, Q \in \mathcal{P}$ from different orbits under $T$. (Here $T_{P}$ denotes the stabilizer $\{\tau \in T \mid \tau(P)=P\}$ of $P$ in $T$.)

$T$ is called planar, if, in addition,

(4) $T=T_{P} \cdot T_{Q}$ and $T_{P} \cap T_{Q}=1$ for suitable $P, Q \in \mathcal{P}$ with $P, Q$ from different orbits.

(5) For every $\tau \in T$ there exists a $P \in \mathcal{P}$ with $\tau(P)=P$.

As already mentioned, many different constructions of DDs can be found in the literature. We recall three of them, all of which give examples of DDs with a dual translation group. 
(a) A method to construct $t$-DDs uses a finite affine space and its translation group [13]. This method of Schulz and Spera, or slightly varied forms, are used e.g. in [3], [4], [5], [6], [9], [14], [16]. In each of these constructions, a suitable subset $\mathcal{P}$ of affine hyperplanes is considered a point set and the point classes are induced by the parallelism relation given in the affine space. After choosing a suitable transversal subset of $\mathcal{P}$, called base block, the block set $\mathcal{B}$ is determined to be the orbit of the base block under a permutation group which contains the translation group and operates $t$-transitively on the set of all transversal $t$-tuples consisting of elements of $\mathcal{P}$. By a proposition of A.G. Spera, this gives a $t$-DD [15].

The permutation group used for constructing the $t$-DD can be considered a (point- and block-transitive) automorphism group ([13], Prop. 2.3 . Since it contains a subgroup induced by the translation group of the affine space fulfilling 2.2, every $t$-DD obtained by this method admits an elementary abelian dual translation group [6]. It fulfills (4) if the affine space is 2-dimensional.

(b) In [6] (see also [8]), a construction of DDs is presented which extends the method of Schulz and Spera. Here, we need a so-called starter design, instead of a base block. This method can be applied to any $t$ DD. We consider an isomorphic image of the starter design whose point set consists of affine hyperplanes all containing a distinguished affine point called the origin. Uniting all its translates gives a $t$-DD ([6], Th. 3.2.4) which in addition is nearly block-decomposable ([6], Remark 3.2.11) (for the concept of block-decomposability see [6], Chapter 2, or [7]). Since the translation group of the affine space operates on the point and block set of the constructed $t$-DD as an automorphism group and all conditions of Definition 2.2 (1) - (3) are fulfilled, it can be considered an (elementary abelian) dual translation group.

(c) In [2], A. Blunck, H. Havlicek and C. Zanella introduce a generalized construction. Again, a starter design - the authors name it base design - is used to get a larger $t$-DD by a so-called t-lifting ([2], Th. 2.5). They also use the properties of a group acting on a suitable finite set of points. The special situation of a $t$-DD arising from point sets in a finite projective or affine space is presented. In ([2], Th. 3.1), they show sufficient properties of the elements of the starter design to get a series of lifted $t$-DDs by using a group $G$ which is elementary abelian and yields an elementary abelian dual translation group of the lifted $t$-DD (cp. [2], 3.12). 


\section{Characterizing some $t$-DDs}

It is not very surprising that the $t$-DDs discussed in the previous part admit dual translation groups, since all of them arise in some manner from finite affine spaces. More interesting is, vice versa, the following.

Theorem 3.1 Let $\mathcal{D}$ be a (non-trivial) divisible design on which there operates an elementary abelian group $\tilde{T}$ inducing a full dual translation group $T$ of $\mathcal{D}$.

(a) Then $\mathcal{D}$ is isomorphic to a divisible design $\hat{\mathcal{D}}$ whose points are (some) affine subspaces of the same dimension of an affine space $\mathcal{A}$ and whose point classes are unions of full parallel classes; and $T$ is induced by the translation group of $\mathcal{A}$.

(b) If, in addition, $T$ is planar then $\mathcal{A}$ can be considered as a translation plane, the points of $\hat{\mathcal{D}}$ as the lines of $\mathcal{A}$, the point classes as unions of full parallel classes of $\mathcal{A}$ and $T$ as the full translation group of $\mathcal{A}$.

Proof: $A d(a)$ : From the group $T$, we are going to construct a geometry $\hat{\mathcal{D}}$ consisting of affine subspaces of an affine space $\mathcal{A}$; then, we show that this geometry is isomorphic to $\mathcal{D}$, and $T$ is induced by the translation group of $\mathcal{A}$. Finally, we shall deduce some properties of $\hat{\mathcal{D}}$.

Let $\mathcal{D}=(\mathcal{P}, \mathcal{B}, \mathcal{S})$ be a (non-trivial) finite DD admitting a dual translation group $T$ induced by an elementary abelian group $\tilde{T}$. As homomorphic image of $\tilde{T}$, the group $T$ is elementary abelian, too. Choose a point $P_{O}$ from each orbit of $\mathcal{P}$ and let $\hat{O}$ be the set of these chosen points. Now, we define

$$
\hat{G}:=\left\{\tau T_{P_{O}} \mid \tau \in T \text { and } P_{O} \in \hat{O}\right\} .
$$

It is well known that an elementary abelian group $T$ of exponent $p$ can be interpreted as a vector space over the field $\operatorname{GF}(p)$. So, the elements of $\hat{G}$ are affine subspaces of a finite affine space $\mathcal{A}$. By definition of $\hat{O}$, for every $P \in \mathcal{P}$ there exists a $\tau_{1} \in T$ and a $P_{O} \in \hat{O}$ such that $\tau_{1}\left(P_{O}\right)=P$. Define $\bar{P}=\overline{\tau_{1}\left(P_{O}\right)}:=\tau_{1} T_{P_{O}}$; then $\bar{P} \in \hat{G}$. The mapping

$$
\rho: \mathcal{P} \rightarrow \hat{G} \text { with } P \mapsto \bar{P}
$$

is well defined and surjective. If $\bar{P}=\tau_{1} T_{P_{O}}=\tau_{2} T_{Q_{O}}=\bar{Q}$, then $T_{P_{O}}=T_{Q_{O}}$, therefore $P_{O}=Q_{O}$ by (3) (of definition (2.2)) and $P=\tau_{1}\left(P_{O}\right)=\tau_{2}\left(Q_{O}\right)=$ $Q$. Hence, $\rho$ is a bijection. For $\tau_{1} \in T$, define $\hat{\tau}_{1}$ by $\hat{\tau}_{1}(\tau):=\tau_{1} \tau$. It is well known that $\hat{\tau}_{1}$ is a translation of $\mathcal{A}$ and $\hat{T}=\{\hat{\tau} \mid \tau \in T\}$ can be seen as the (full) translation group of $\mathcal{A}$. The elements of $\hat{G}$ are permuted among themselves and the orbits under $\hat{T}$ are full parallel classes.

The mapping $\rho$ is compatible with the actions of $T$ on $\mathcal{P}$ and of $\hat{T}$ on $\hat{G}$ since $\rho \tau(P)=\overline{\tau(P)}=\overline{\tau \tau_{1}\left(P_{O}\right)}=\tau \tau_{1} T_{P_{O}}=\hat{\tau} \rho(P)$ for all $P \in \mathcal{P}$. The 
bijection $\rho$ thus gives rise to an isomorphism of group actions and maps blocks onto corresponding subsets. So, similar to the situation of [1] and [17], our geometry can be reconstructed from its automorphism group. In particular, it follows that $\hat{T}$ operates faithfully on $\hat{G}$.

We want to show that all elements of $\hat{G}$ have the same dimension. Let $P_{O} \in \hat{O}$. By $|T|=\left|T_{P_{O}}\right| \cdot\left|P_{O}^{T}\right|$, and the assumption about the length of the orbit, we have $\left|\overline{P_{O}}\right|=\left|T_{P_{O}}\right|=|T| /\left|P_{O}^{T}\right|=|T| / l$ for all $P_{O} \in \hat{O}$. If $|T| / l=p^{t}$, every element of $\hat{G}$ has dimension $t$ over $\operatorname{GF}(p)$.

$A d(b)$ : Now, let $T$ be planar; by part (a) $\mathcal{D}$, is isomorphic to a DD $\hat{\mathcal{D}}$ whose points are affine subspaces of the same dimension and whose point classes are unions of full parallel classes of a finite affine space $\mathcal{A}$, and $T$ can be considered as the translation group of $\mathcal{A}$.

By condition (4), the intersection of two elements of $\left\{T_{P_{O}} \mid P_{O} \in \hat{O}\right\}$ is always equal to 1 , an element which can be considered as the origin; by condition (5), every $\tau \in T$ fixes an element $P=\tau_{1}\left(P_{O}\right) \in \mathcal{P}$ (with $P_{O} \in \hat{O}$ and $\tau_{1} \in T$ suitable), and by $\tau_{1}\left(P_{O}\right)=P=\tau(P)=\tau \tau_{1}\left(P_{O}\right)=\tau_{1} \tau\left(P_{O}\right)$ this also holds for $P_{O}$. This gives $\tau \in T_{P_{O}}$. Thus, the set $\left\{T_{P_{O}} \mid P_{O} \in \hat{O}\right\}$ is a non-trivial partition of $T$ and, with condition (4), a spread of $T$.

By J. André [1] (see also [10], Th. 1.4) $T$ can be considered a translation plane $\mathcal{A}, \hat{G}$ the set of lines of $\mathcal{A}$ through the origin, point classes the unions of parallel classes and $T$ the full translation group of $\mathcal{A}$.

We would like to thank the referees for useful comments.

\section{References}

[1] J. André. Über nicht-Desarguessche Ebenen mit transitiver Translationsgruppe. Math. Z., 60:156-186, 1954.

[2] Havlicek H. Blunck, A. and C. Zanella. Lifting of divisible designs. Des. Codes Cryptography, 42:1-14, 2007.

[3] C. Cerroni and R.-H. Schulz. Divisible designs admitting GL $(3, q)$ as an automorphism group. Geometriae Dedicata, 83:343-350, 2000.

[4] C. Cerroni and R.-H. Schulz. Divisible designs admitting, as an automorphism group, an orthogonal group or a unitary group. In : Dieter Jungnickel et al., editors, Finite fields and applications, pages 95-108. Proc. Fifth. Internat. Conf. on Finite Fields and Applications, SpringerVerlag, Berlin, 2001.

[5] C. Cerroni and A.G. Spera. On divisible designs and twisted field planes. J. Combin. Designs, 7:453-464, 1999.

[6] S. Giese. Block-zerlegbare divisible Designs. URL: http://www.diss.fuberlin.de/2005/347, 2005. Dissertation. 
[7] S. Giese. Block-decomposability of divisible designs. Submitted to J.Comb.Des., 2006.

[8] S. Giese. Constructing block-decomposable divisible designs. Preprint, 2006.

[9] S. Giese, H. Havlicek, and R.-H. Schulz. Some constructions of divisible designs from Laguerre geometries. Discrete Mathematics, 301:74-82, 2005.

[10] H. Lüneburg. Translation Planes. Springer-Verlag, New York, 1980.

[11] R.-H. Schulz. On the classification of translation group-divisible designs. Europ. J. Combin., 6:369-374, 1985.

[12] R.-H. Schulz. On translation transversal designs with $\lambda>1$. Arch. Math., 49:97-102, 1987.

[13] R.-H. Schulz and A.G. Spera. Construction of divisible designs from translation planes. Europ. J. Combin., 19(4):479-486, 1998.

[14] R.-H. Schulz and A.G. Spera. Divisible designs admitting a Suzuki group as an automorphism group. Boll. Unione Mat. Ital., (8)-1-B(No. 3):705-714, 1998.

[15] A.G. Spera. $t$-Divisible designs from imprimitive permutation groups. Europ. J. Combin., 13:409-417, 1992.

[16] A.G. Spera. Divisible designs associated with translation planes admitting a 2-transitive collineation group on the points at infinity. Aequationes Math., 59(1-2):191-200, 2000.

[17] M. Stroppel. Reconstruction of incidence geometries from groups of automorphisms. Arch. Math., 58:621-624, 1992.

Authors' e-mail addresses:

giese@math.fu-berlin.de

schulz@math.fu-berlin.de 